AperTO - Archivio Istituzionale Open Access dell'Università di Torino

\title{
Characterization of bacterial communities of donkey milk by high-throughput sequencing
}

\section{This is the author's manuscript}

Original Citation:

Availability:

This version is available http://hdl.handle.net/2318/1635221

since 2019-02-18T15:28:53Z

Published version:

DOI:10.1016/j.ijfoodmicro.2017.03.023

Terms of use:

Open Access

Anyone can freely access the full text of works made available as "Open Access". Works made available under a Creative Commons license can be used according to the terms and conditions of said license. Use of all other works requires consent of the right holder (author or publisher) if not exempted from copyright protection by the applicable law. 
1 Characterization of bacterial communities of donkey milk by high-throughput

2 sequencing.

3

4 Maria de los Dolores Soto del Rio ${ }^{a}$, Alessandra Dalmasso ${ }^{\mathrm{a}^{*}}$, Tiziana Civera ${ }^{\mathrm{a}}$, Maria Teresa 5 Bottero $^{\mathrm{a}}$

6

7 a Dipartimento di Scienze Veterinarie, Università di Torino, Largo Braccini 2, 10095 8 Grugliasco (TO), Italy. mariadelosdolores.sotodelrio@ unito.it, alessandra.dalmasso@ unito.it,

9 tiziana.civera@unito.it, mariateresa.bottero@unito.it

10

* Corresponding author.

Tel.: +390116709215

Fax: +39 0116709224

Email address: alessandra.dalmasso@unito.it,

The authors declare no conflict of interest.

All authors participated in both research and manuscript preparation. All authors have approved the final version of this article.

The research has been supported by a grant of the University of Turin (ex 60\%). 


\section{Abstract}

The interest in donkey milk (DM) is growing because of its functional properties and nutritional value, especially for children with allergies and food intolerances. However, most of the available reports of DM microbiota are based on culture-dependent methods to investigate food safety issues and the presence of lactic acid bacteria (LAB).

The aim of this study was to determine the composition of DM bacterial communities using a high-throughput sequencing (HTS) approach.

Bulk milk samples from Italian donkey dairy farms from two consecutive years were analysed using the MiSeq Illumina platform. All sample reads were classified into five phyla: Proteobacteria, Firmicutes, Bacteroidetes, Actinobacteria, and Verrucomicrobia. The most prevalent genera-Pseudomonas, Ralstonia, Acinetobacter, Cupriavidus, Citrobacter and Sphingobacterium - were gram-negative bacteria.

The core microbiota was composed of genera that comprise commonly associated milk bacteria, LAB and species normally found in soil, water and plants. Reads assigned to LAB genera-Streptococcus, Lactococcus, Enterococcus, Leuconostoc, Lactobacillus, and Carnobacterium - corresponded on average to $2.55 \%$ of the total reads per sample. Among these, the distribution of reads assigned to coccus- and bacillus-shaped LAB was variable between and within the farms, confirming their presence and suggesting a complex population of these bacteria in DM.

The present study represents a general snapshot of the DM microbial population, underlining its variability and motivating further studies for the exploitation of the technological potential of bacteria naturally present in DM. 
Keywords:

donkey milk, bacterial communities, high-throughput sequencing

54

Highlights:

56 Bulk milk samples of donkey milk were studied with a HTS approach.

57 Microbial population of DM is complex, diverse, variable

The most prevalent genera are Gram negative bacteria.

59 


\section{Introduction}

Donkey milk (DM) has recently received growing interest since it has been reported to be an adequate replacement for children with cow milk protein allergy, mainly due to its tolerability, nutritional contents and good taste (Monti et al., 2012). In fact, studies have demonstrated a number of qualities that make DM more favourable than cow milk: better digestibility (Tidona et al., 2011), lower allergenicity (Vincenzetti et al., 2008) and a set of unique nutritional and physicochemical characteristics (Guo et al., 2007).

Following the growing demand for DM, several new dairy farms have opened in the last few years. Italian donkey dairies are generally small, with 20 to 25 milking jennies and one or two stallions; their overall average daily production is approximately 2,000 litres, for a total of 700,000 litres per year (Milonis and Polidori, 2011). The production is mainly used for direct human consumption, while a smaller part is destined for the cosmetics and food industries. Pasteurized donkey milk is usually sold directly from the farms. However, considering its target consumers and nutritional properties, it can be sold raw, with 3 days of shelf life (similar to raw bovine milk) (Giacometti et al., 2016).

The composition of DM is closer to human milk than to cow milk and has been fully described (Salimei and Fantuz, 2012). It contains high levels of lactose and essential amino acids (Guo et al., 2007) as well as low concentrations of $\beta$-lactoglobulin and casein - the most common allergens in cow milk (Vincenzetti et al., 2008). One of the main characteristics of DM is its high concentration of lysozyme: from 1300 to $4000 \mathrm{mg} / \mathrm{l}$, compared to $0.09 \mathrm{mg} / \mathrm{l}$ in cow milk and 40-200 mg/l in human milk (Carminati et al., 2014; Chiavari et al., 2005; Vincenzetti et al., 2008). This enzyme has bactericidal properties; it hydrolyses the murein of bacterial cell walls, causing lysis of sensitive bacteria (Chiavari et al., 2005). Currently, there is no confirmed hypothesis as to why DM is so rich in lysozyme, but it seems to positively affect the animals, defending against infections in both the mammary gland and the foal. In 
addition to lysozyme, DM lactoferrin concentration is twice as high as in bovine milk (Malacarne et al., 2002), and other components have been described, such as immunoglobulins, free fatty acids and members of the lactoperoxidase peroxide system (Zhang et al., 2008), that might act synergistically against specific bacteria (Šarić et al., 2012). Traditional microbiological tests and biomolecular culture-dependent methods have been used to study the bacterial population of DM, mainly focusing on hygienic conditions and/or the presence of lactic acid bacteria (LAB) (Cavallarin et al., 2015; Pilla et al., 2010; Zhang et al., 2008; Šarić et al., 2012). Moreover, in the last few years, culture-independent methods, based on the direct analysis of DNA without a culturing step, have also been used to characterize the milk of different species (Quigley et al., 2013). PCR-denaturing gradient gel electrophoresis (PCR-DGGE), for example, has been successfully applied to the study of the microbiota of milk and dairy products (Delgado et al., 2013). However, limitations in the resolution still need to be overcome, especially for the analyses of matrices with diverse microbial communities (Ogier et al., 2004). Recently, rapid developments of high-throughput sequencing (HTS) methods have allowed a deeper and more precise evaluation of the milk microbiota from different animals, including cattle, goat, sheep, buffalo and humans (Quigley et al., 2013).

Notwithstanding the extensive literature on DM, no high-throughput analysis of its bacterial population has yet been performed, despite ever-increasing interest from both technological and commercial points of view. For this reason, the present study aimed to contribute to the knowledge of DM by characterizing its microbiota using an HTS approach.

\section{Materials and Methods}

\subsection{Milk sampling and DNA extraction}


110 Five donkey dairy farms $(\mathrm{A}, \mathrm{B}, \mathrm{C}, \mathrm{D}, \mathrm{E})$ in the northwest part of Italy were sampled during the spring (March) of 2013 (samples A.2013, B.2013, C.2013, D.2013, E.2013) and 2014 (samples A.2014, B.2014, C.2014, D.2014, E.2014); in the second year, an additional farm was included (F; sample F.2014). These are small dairies, with a few milking jennies, familyrun and with a limited production (around one litre per day, per animal); the general characteristics of the surveyed farms are summarized in Table S1. The biochemical characterization, the shelf life and the safety of the samples have been reported in a previous work (Cavallarin et al., 2015).

Bulk milk samples from healthy jennies, collected in sterile tubes, were transported to the laboratory immediately after sampling in cool conditions and stored at $-20{ }^{\circ} \mathrm{C}$ until DNA extraction. Samples were treated as reported elsewhere (Dalmasso et al., 2011), and DNA was extracted from $3 \mathrm{ml}$ of milk following the manufacturer protocol of the Dneasy Blood \& Tissue kit (Qiagen) and quantified with a Nanodrop 2000 (Thermo Fisher Scientific). To minimize the bias associated with single extractions, triple extractions of each sample were done in parallel and mixed in a final pool.

\subsection{High-throughput sequencing}

Illumina libraries were prepared following the protocol described by Dalmasso et al. (2016) with the NEXTflex 16S V4 Amplicon-Seq Kit (Bioo Scientific, Austin, USA). Briefly, the bacterial V4 region of the $16 \mathrm{~S}$ ribosomal gene was amplified from $50 \mathrm{ng}$ of DNA for each sample. The universal primers $515 \mathrm{~F}$ and $806 \mathrm{R}$ tailed with Illumina barcoded adapters were used with the following touchdown PCR conditions: an initial 9 cycles $\left(15 \mathrm{sec}\right.$. at $95^{\circ} \mathrm{C}, 15$ sec. at $68^{\circ} \mathrm{C}, 30 \mathrm{sec}$. at $\left.72^{\circ} \mathrm{C}\right)$ and then another 23 cycles $\left(15 \mathrm{sec}\right.$. at $95^{\circ} \mathrm{C}, 15 \mathrm{sec}$. at $58^{\circ} \mathrm{C}, 30$ sec. at $72^{\circ} \mathrm{C}$ ). The PCR products were purified using Agencourt XP Ampure Beads (Beckman 
Coulter). The quality of the final products was assessed with a Bioanalyzer 2100 (Agilent Technologies).

The samples were quantified with Qubit (Invitrogen) and pooled in equal proportions for their paired-end sequencing with Illumina MiSeq for 312 cycles (150 cycles for each paired read and 12 cycles for the barcode sequence) at IGA Technology Services (Udine, Italy). To prevent focusing and phasing problems due to the sequencing of "low diversity" libraries, $30 \%$ PhiX genome was spiked in the pooled library.

\subsection{Bioinformatics and data analyses}

Sequence reads were trimmed with the collection command line tools of FASTX-Toolkits (http://hannonlab.cshl.edu/fastx_toolkit/) so that the quality score for each read was above 20 with more than 50 base pairs. The PRINSEQ standalone lite version (Schmieder and Edwards, 2011) was used to check and prepare the data set for the downstream analyses.

Data were then analysed with the QIIME software, version 1.9.0 (Caporaso et al., 2012). Using the uclust method (Edgar, 2010), sequences >97\% identical were considered to correspond to the same operational taxonomic unit (OTU). Representative sequences were submitted to the RDPII classifier (Wang et al., 2007) to obtain the taxonomy assignment and relative abundance of each OTU using the Greengenes 16S rDNA database v13.8 (McDonald et al., 2012).

Alpha diversity was evaluated with QIIME to obtain the rarefaction curves. A rarefaction curve shows the variation in the number of OTUs identified at a given percentage of identity as a function of the number of sequence reads obtained per sample. Ideally, an optimal coverage is identified by the plateau of the curve, which indicates that increasing the number of reads does not change the number of OTUs that can be determined. 
Moreover, Good's coverage (a sampling completeness indicator that indicates what percent of the total species is represented in the sample), Chao1 and ACE (richness estimators that calculate an approximate number of species in the samples using different methods), and Shannon and Simpson indices (estimators of the samples' diversity taking into account the approximated number of species and how evenly they are distributed) were determined.

Beta diversity was evaluated with the UniFrac method. Weighted UniFrac distance matrices and OTU tables were used to plot the principal coordinate analysis (PCoA) and to perform Adonis and Anosim statistical tests with the compare_category.py script of QIIME to evaluate differences between the farms, their practices and their characteristics.

The core microbiota of the samples was obtained with the compute_core_microbiome.py script in QIIME; OTUs present with more than $0.001 \%$ of the reads of each sample, in at least 9 samples, were included. The pseudo-heatmap was plotted with the gplots package in the $\mathrm{R}$ environment (http://www.r-project.org) using the OTUs table generated by QIIME.

\section{Results and Discussion}

\subsection{Characteristics of the sequencing data}

We obtained a total of 5,225,689 raw sequences; after filtering, 3,743,291 high-quality $16 \mathrm{~S}$ rRNA gene sequences with an average length of 288 bp were recovered. Table 1 shows the number of analysed reads per sample. The rarefaction curves of our data (Figure S1) suggest a sufficient coverage; this consideration is further supported by the observed values of the Good's coverage estimator -higher than 0.99- for all the samples (Table 1).

\subsection{Bacterial composition of donkey milk}

The sequences obtained from all the studied samples correspond to five phyla: Proteobacteria, Firmicutes, Bacteroidetes, Actinobacteria and Verrucomicrobia (Table 2) in 
agreement with the main taxons found in raw milk from different animals (Dalmasso et al. 2016; Quigley et al., 2013). The total reads corresponded to 201 families and 314 different genera (data not shown).

The most abundant genera observed in all the studied samples were gram-negative bacteria: Pseudomonas, Ralstonia, Cupriavidus, Acinetobacter, Citrobacter and Sphingobacterium (Figure 1, Table 2).

However, only the genus Pseudomonas reached high percentages in almost all the studied samples. Furthermore, previous studies, using culture-dependent methods, had found that Pseudomonas spp. is an important component of the DM microbiota (Cavallarin et al., 2015; Giacometti et al., 2016). This observation is consistent with a previous report that indicated Pseudomonas spp. to be the predominant microorganism in different milks (Quigley et al., 2013); in raw bovine milk stored at low temperatures, Pseudomonas spp. may constitute up to $70-90 \%$ of the total microbial population (Sørhaug and Stepaniak, 1997). The abundance of these microorganisms, which are the most common cause of milk spoilage (Ercolini et al., 2009), mainly because of their proteolytic activity and psychrotolerant nature, leads to the short commercial shelf life of the product ( 3 days). Given that raw DM is sold, is necessary to focus attention not only on spoilage but also on hygienic safety. Cavallarin et al., (2015), while characterizing DM by traditional microbiological methods, showed the absence of pathogens. In our study, the limitations of the analytical approach (genus identification and the impossibility of viability evaluation) did not allow us to infer the hygienic safety status. The other genera (Ralstonia, Cupriavidus, Acinetobacter, Citrobacter and Sphingobacterium) (Figure 1, Table 2), are considered environmental microorganisms since they are commonly found in soil, water and dust. Ralstonia spp. and Cupriavidus spp. are phylogenetically related to Pseudomonas spp., and they have only recently been reclassified (Balkwill, 2015; 
Yabuuchi et al., 2015). Nevertheless, HTS studies have found them in human, bovine, goat and buffalo milk (Quigley et al., 2013).

The composition of the DM core microbiota, i.e., those OTUs shared between the samples, was also evaluated. This core contained 4 families and 24 genera that comprise commonly associated milk bacteria, LAB and species normally found in soil, water and plants (Figure 2). One compelling member of the core was the genus Akkermansia since the only species that currently forms the genus, Akkermansia muciniphila, has been linked with intestinal health, the metabolic status of obese and diabetic patients, and markers of inflammation and immune responses (Reunanen et al., 2015). This potential probiotic bacterium uses mucin - a protein amply present in milk - as its main source of carbon and nitrogen and has been detected in human and animal gut environments (Belzer and de Vos, 2012), including in donkeys (Liu et al., 2014). Additionally, this bacterium has been detected in breast milk using real-time PCR (Collado et al., 2012), and just recently, Ottman (2015) reported its ability to grow in human milk. Further studies are needed to isolate and characterize the probable Akkermansia species present in DM; nonetheless, our observation creates a new perspective on this functional microbe that has not yet been isolated from food matrices.

Subsequently, we analysed the differences in the distribution of the OTUs between and within the farms, where some particular trends were observed. Beta diversity analyses, using the UniFrac method, were performed to compare the samples between the dairies. We performed Anosim and Adonis tests for all the different parameters of the dairies (farm area, altitude of the farm, breed, milking practice, farming type and feeding), but none of them had a significant $(\mathrm{P}>0.01)$ influence on the variation observed in the DM microbiota (data not shown). The only variable that resulted in significant differences was the sampling year, indicating that the bacteria present in the samples from 2013 were different from those from 2014 (Figure S2 of the supplementary material). This very interesting result suggests that the 
variability in the milk microbiota may derive from the individual components of each animal and/or their lactation period. As the gestation period in donkeys is approximately one year and jennies produce milk only for 6 months, we sampled milk from completely different animals in each year. Moreover, the different stages of lactation of the milking jennies in each farm would further contribute to the variability observed. These interindividual differences have been amply described for breast milk (Cabrera-Rubio et al., 2012), and we can most likely assume that they are also valid for other mammal milks; still, further studies are needed to corroborate this presumption.

Additionally, the Chao1 richness estimator and the Shannon diversity index of Farm D (Table 1) and its rarefaction curves (Figure S1) demonstrated that this farm had the fewest number of observed genera of all the tested farms. In particular, the most representative were Ralstonia and Cupriavidus spp. (Figure 1). This low variability could be a consequence of the farming practices since it is the only sampled farm run extensively; the animals are free to pasture and are hand milked only when it is requested (Table S1). Moreover, Cavallarin et al. (2015) showed that the samples from this dairy had lower total bacterial counts than those milked automatically. This thesis could be further confirmed by i) the higher percentage of Streptococcus spp. reads (Figure 3A), a genus considered skin-associated (Cogen et al., 2007), and ii) the low percentage of Pseudomonas spp. reads (Figure 3B); members of this genus are normally present in water, and they might derive from the water used to rinse the milking machinery. The supposition that farm practices have a direct consequence in the milk microbiota has also been supported by goat farm observations, where hand milking practices resulted in lower total bacteria counts (Delgado-Pertiñez et al., 2003).

\subsection{Lactic acid bacteria in donkey milk}


Studies regarding the microbiota of DM have focused on the hygienic quality of DM (Pilla et al., 2010; Zhang et al., 2008; Šarić et al., 2012). Only more recently have some authors characterized the lactic bacteria for their probiotic activity and potential technological aspects (Carminati et al., 2014; Soto del Rio et al., 2016). It is generally accepted that LAB are the dominant population in milk from several species, independent of the methodology used for study. Reports with an HTS approach in cow, sheep, buffalo and human milk have identified LAB reads that corresponded to more than $40 \%$ of the total sequences (Quigley et al., 2013). In our samples, we detected reads for the LAB genera Carnobacterium, Enterococcus, Lactobacillus, Lactococcus, Leuconostoc and Streptococcus (Figure 3A, Table 2) with an average of $2.55 \%$, ranging from $0.02 \%$ (zoomed in Figure 3B) to $15.85 \%$, of the total reads per sample, which is consistent with the low LAB count in these samples reported by

\section{Cavallarin et al., (2015).}

In this study, all the samples had sequences that corresponded to both coccus (Enterococcus, Lactococcus, and Streptococcus)- and bacillus (Carnobacterium, Lactobacillus, and Leuconostoc)-shaped genera (Figure 3), although in different proportions. This result is in contrast with other studies, where the authors isolated and characterized only coccus-shaped LAB (Carminati et al., 2014) or bacilli species (Soto del Rio et al., 2016). However, there was important variability in the distribution of cocci/bacilli reads both within and between the different farms (Figure 3). In particular, cocci were noticeably present only in Farms C and E in both sampling years (Figure 3C), whereas sample D.2013 presented more cocci reads. Sample A.2014 was characterized by a similar proportion of bacilli and cocci reads. Regarding the bacilli, the sole sampling year of Farm F showed only bacilli reads (Figure 3C), while in Farm B, their presence was not constant; in 2013, the prevalence of cocci was clear, while the situation was reversed in the following year. It is relevant to note that these two 
bacilli-rich samples (B.2014 and F.2014) were the ones that had higher percentages of LAB reads from the total number of sequences (Figure 3A).

These results are relevant to the possible production of probiotic milks. Several authors have proposed novel fermented DM beverages that used lactobacilli strains isolated from bovine milk adapted to grow in DM (Chiavari et al., 2005; Perna et al., 2015). Consequently, having available bacilli strains naturally adapted to DM might be notable from a biotechnological point of view to facilitate the production of these beverages.

Overall, the results suggest that the LAB population of DM is complex, diverse, variable and may depend upon several parameters, thus requiring further investigation.

\section{Conclusions}

The present survey provides a broad characterization of the bacterial composition of DM, allowing a description of microorganisms not previously detected in this product. The microbiota of DM is mainly composed of gram-negative bacteria. Unlike other milks, LAB reads were present in low percentages, both cocci and bacilli, even though their growth is not particularly favoured by the composition of DM. The HTS analysis of diverse farms allowed the proposal of several genera as members of a core DM microbiota. The observed results also support the premise that the microbial composition of DM may be influenced by individual animal components.

The present study aimed to give a general picture of the bacterial communities present in DM, and it has shown that this microbiota can be highly diverse. Further studies are needed to better understand the dynamics between the bacterial population in this matrix and the relationship between the milk components. 
The authors would like to thank the owners of the Piedmont donkey milk farms for their availability and cooperation in the sampling. total number of reads per sample.

Figure 1. Distribution of the most abundant genera in donkey milk. Percentages refer to the

Figure 2. Core microbiota of donkey milk. A) Taxonomic distribution of the OTUs present at $>0.001 \%$ in at least nine samples. B) Pseudo-heatmap of the distribution (\%) of the core OTUs. Samples were clustered using Euclidean distance and the complete method.

Figure 3. Distribution of lactic acid bacteria detected in donkey milk samples. A) Abundance of LAB genera found in the studied samples; percentages refer to the total number of reads. B) Zoomed-in for the lower percent levels of LAB genera abundance in each sample C) Relative abundance for the sum of the percentages of coccus-shaped (Enterococcus, Lactococcus, Streptococcus) and bacillus-shaped (Carnobacterium, Lactobacillus, Leuconostoc) LAB genera reads for each farm.

Table 1. Numbers of sequences analyzed, observed OTUs, coverage and diversity estimators for all the studied samples.

Table 2. Percentages of the most abundant taxonomical groups of the sampled donkey milk farms.

Figure S1. Rarefaction curves of the observed species for each studied sample.

Figure S2. Principal coordinate analysis (PCoA) of the surveyed donkey milk samples. The plot was based on the weighted UniFrac distance matrix of the microbiota. The dots and names in red correspond to the sampling of 2013, while the blue ones correspond to 2014. 
Table S1. General characteristics of the surveyed donkey milk farms. Modified from (Cavallarin et al., 2015)

\section{References}

Balkwill, D.L. 2015. Cupriavidus. Bergey's Manual of Systematics of Archaea and Bacteria. $1-7$.

Belzer, C., de Vos, W.M., 2012. Microbes inside-from diversity to function: the case of Akkermansia. ISME J. 6, 1449-1458.

Cabrera-Rubio, R., Collado, M.C., Laitinen, K., Salminen, S., Isolauri, E., Mira, A., 2012. The human milk microbiome changes over lactation and is shaped by maternal weight and mode of delivery. Am. J. Clin. Nutr. 96, 544-551.

Caporaso, J.G., Lauber, C.L., Walters, W.A., Berg-Lyons, D., Huntley, J., Fierer, N., Owens, S.M., Betley, J., Fraser, L., Bauer, M., Gormley, N., Gilbert, J.A., Smith, G., Knight, R., 2012. Ultra-high-throughput microbial community analysis on the Illumina HiSeq and MiSeq platforms. ISME J. 6, 1621-1624.

Carminati, D., Tidona, F., Fornasari, M.E., Rossetti, L., Meucci, A., Giraffa, G., 2014. Biotyping of cultivable lactic acid bacteria isolated from donkey milk. Lett. Appl. Microbiol. 59, 299-305.

Cavallarin, L., Giribaldi, M., Soto-Del Rio, M.D., Valle, E., Barbarino, G., Gennero, M.S., Civera, T., 2015. A survey on the milk chemical and microbiological quality in dairy donkey farms located in NorthWestern Italy. Food Control 50, 230-235. 
Chiavari, C., Coloretti, F., Nanni, M., Sorrentino, E., Grazia, L., 2005. Use of donkey's milk for a fermented beverage with lactobacilli. Lait 85, 481-490.

Collado, M.C., Laitinen, K., Salminen, S., Isolauri, E., 2012. Maternal weight and excessive weight gain during pregnancy modify the immunomodulatory potential of breast milk. Pediatr. Res. 72, 77-85.

Cogen, A.J., Nizet, V., Gallo, R.L., 2007. Skin microbiota: a source of disease or defence? Brit. J. Dermatol. 158, 442-455.

Dalmasso, A., Civera, T., La Neve, F., Bottero, M.T., 2011. Simultaneous detection of cow and buffalo milk in mozzarella cheese by Real-Time PCR assay. Food Chem. 124, 362-366.

Dalmasso, A., Soto del Rio, MDLD., Civera, T., Pattono, D., Cardazzo, B., Bottero, MT., 2016. Characterization of microbiota in Plaisentif cheese by high-throughput sequencing. LWT-Food Sci. Technol. 69, 490-496.

Delgado, S., Rachid, C.T., Fernández, E., Rychlik, T., Alegría, Á., Peixoto, R.S., Mayo, B., 2013. Diversity of thermophilic bacteria in raw, pasteurized and selectively-cultured milk, as assessed by culturing, PCR-DGGE and pyrosequencing. Food Microbiol. 36, 103-111.

Delgado-Pertiñez, M., Alcalde, M.J., Guzmán-Guerrero, J.L., Castel, J.M., Mena, Y., Caravaca, F., 2003. Effect of hygiene-sanitary management on goat milk quality in semiextensive systems in Spain. Small Ruminant Res. 47, 51-61.

Edgar, R.C., 2010. Search and clustering orders of magnitude faster than BLAST. Bioinformatics 26, 2460-2461. 
Ercolini, D., Russo, F., Ferrocino, I., Villani, F., 2009. Molecular identification of mesophilic and psychrotrophic bacteria from raw cow's milk. Food Microbiol. 26, 228-231.

Giacometti, F., Bardasi, L., Merialdi, G., Morbarigazzi, M., Federici, S., Piva, S., Serraino, A., 2016. Shelf life of donkey milk subjected to different treatment and storage conditions. J. Dairy Sci. 99, 1-9.

Guo, H.Y., Pang, K., Zhang, X.Y., Zhao, L., Chen, S.W., Dong, M.L., Ren, F.Z., 2007. Composition, physiochemical properties, nitrogen fraction distribution, and amino acid profile of donkey milk. J. Dairy Sci. 90, 1635-1643.

Liu, X., Fan, H., Ding, X., Hong, Z., Nei, Y., Liu, Z., Li, G., Guo, H., 2014. Analysis of the gut microbiota by high-throughput sequencing of the v5-v6 regions of the 16s rRNA gene in donkey. Curr. Microbiol. 68, 657-662.

Malacarne, M., Martuzzi, F., Summer, A., Mariani, P., 2002. Protein and fat composition of mare's milk: Some nutritional remarks with reference to human and cow's milk. Int. Dairy J. $12,869-877$.

McDonald, D., Price, M.N., Goodrich, J., Nawrocki, E.P., Desantis, T.Z., Probst, A., Andersen, G.L., Knight, R., Hugenholtz, P., 2012. An improved Greengenes taxonomy with explicit ranks for ecological and evolutionary analyses of bacteria and archaea. ISME J. 6, $610-618$.

Milonis, E., Polidori, P., 2011. Latte di asina: produzione, caratteristiche e gestione dell'azienda asinina, Fondazione Iniziative Zooprofilattiche e Zootecniche, Brescia, Italia. 
Monti, G., Viola, S., Baro, C., Cresi, F., Tovo, P.A., Moro, G., Ferrero, M.P., Conti, A., Bertino, E., 2012. Tolerability of donkey's milk in highly-problematic cow's milk allergic children. J. Biol. Reg. Homeos. Ag. 26, 75-82.

Ogier, J.C., Lafarge, V., Girard, V., Rault, A., Maladen, V., Gruss, A., Leveau, J.Y., Delacroix-Buchet, A., 2004. Molecular fingerprinting of dairy microbial ecosystems by use of temporal temperature and denaturing gradient gel electrophoresis. Appl. Environ. Microb. 70, $5628-5643$.

Ottman, N., 2015. Host immunostimulation and substrate utilization of the gut symbiont Akkermansia muciniphila. $\mathrm{PhD}$ thesis, Wageningen University, Wageningen, the Netherlands.

Perna, A., Intaglietta, I., Simonetti, A., Gambacorta, E., 2015. Donkey milk for manufacture of novel functional fermented beverages. J. Food Sci. 80, S1352-S1359.

Pilla, R., Daprà, V., Zecconi, A., Piccinini, R., 2010. Hygienic and health characteristics of donkey milk during a follow-up study. J. Dairy Res, 77, 392-397.

Quigley, L., O'Sullivan, O., Stanton, C., Beresford, T.P., Ross, R.P., Fitzgerald, G.F., Cotter, P.D., 2013. The complex microbiota of raw milk. FEMS Microbiol. Rev. 37, 664-698.

Reunanen, J., Kainulainen, V., Huuskonen, L., Ottman, N., Belzer, C., Huhtinen, H., de Vos, W.M., Satokaria, R., 2015. Akkermansia muciniphila adheres to enterocytes and strengthens the integrity of the epithelial cell layer. Appl. Environ. Microb. 81, 3655-3662.

Salimei, E., Fantuz, F., 2012. Equid milk for human consumption. Int. Dairy J. 24, 146-152.

Schmieder, R., Edwards, R., 2011. Quality control and preprocessing of metagenomic datasets. Bioinformatics. 27, 863-864. 
414 Soto del Rio, M.d.l.D., Andrighetto, C., Dalmasso, A., Lombardi, A., Civera, T., Bottero, 415 M.T., 2016. Isolation and characterisation of lactic acid bacteria from donkey milk. J. Dairy 416 Res, 83, 383-386.

Sørhaug, T., Stepaniak, L., 1997. Psychrotroph and their enzymes in milk and dairy products: Quality aspects. Trends Food Sci. Technol. 8, 35-41.

Tidona, F., Sekse, C., Criscione, A., Jacobsen, M., Bordonaro, S., Marletta, D., Vegarud, G.E., 2011. Antimicrobial effect of donkeys' milk digested in vitro with human gastrointestinal enzymes. Int. Dairy J. 21, 158-165.

Vincenzetti, S., Polidori, P., Mariani, P., Cammertoni, N., Fantuz, F., Vita, A., 2008. Donkey's milk protein fractions characterization. Food Chem. 106, 640-649.

Wang, Q., Garrity, G.M., Tiedje, J.M., Cole, J.R., 2007. Naive Bayesian classifier for rapid assignment of rRNA sequences into the new bacterial taxonomy. Appl. Environ. Microbiol. $73,5261-5267$.

Yabuuchi, E., Kawamura, Y., Ezaki, T. 2015. Ralstonia. Bergey's Manual of Systematics of Archaea and Bacteria. 1-21.

Zhang, X.Y., Zhao, L., Jiang, L., Dong, M.L., Ren, F.Z., 2008. The antimicrobial activity of donkey milk and its microflora changes during storage. Food Control 19, 1191-1195.

Šarić, L.Ć., Šarić, B.M., Mandić, A.I., Torbica, A.M., Tomić, J.M., Cvetković, D.D., Okanović, D.G., 2012. Antibacterial properties of domestic Balkan donkeys' milk. Int. Dairy J. 25, 142-146. 


\begin{tabular}{lccccccc}
\hline Sample & Reads & $\begin{array}{c}\text { Good's } \\
\text { coverage }\end{array}$ & $\begin{array}{c}\text { Observed } \\
\text { OTUs }\end{array}$ & Chao 1 & ACE & Shannon & Simpson \\
\hline A.2013 & 294,557 & 0.994 & 5078 & 6875.19 & 6833.26 & 7.09 & 0.96 \\
A.2014 & 188,349 & 0.993 & 3760 & 5616.95 & 5513.89 & 6.15 & 0.92 \\
B.2013 & 203,091 & 0.993 & 4008 & 5410.35 & 5345.92 & 6.52 & 0.94 \\
B.2014 & 223,728 & 0.993 & 4338 & 6002.83 & 6138.72 & 5.46 & 0.81 \\
C.2013 & 279,374 & 0.993 & 5745 & 7880.04 & 7818.74 & 7.37 & 0.97 \\
C.2014 & 850,529 & 0.998 & 7686 & 9465.44 & 9477.87 & 6.60 & 0.92 \\
D.2013 & 172,717 & 0.996 & 2316 & 2965.35 & 2964.90 & 5.65 & 0.90 \\
D.2014 & 220,559 & 0.997 & 2019 & 2928.77 & 2853.42 & 2.90 & 0.46 \\
E.2013 & 254,323 & 0.994 & 3839 & 5474.76 & 5453.59 & 5.73 & 0.87 \\
\hline E.2014 & 501,861 & 0.997 & 5012 & 6989.73 & 7026.50 & 5.99 & 0.92 \\
\hline F.2014 & 554,203 & 0.997 & 5759 & 7702.27 & 7826.34 & 5.84 & 0.89 \\
\hline
\end{tabular}




\begin{tabular}{|c|c|c|c|c|c|c|c|c|c|c|c|c|}
\hline Phylum & Genus & & & & & & Farms & & & & & \\
\hline & & A.2013 & A. 2014 & B.2013 & B.2014 & C. 2013 & C. 2014 & D.2013 & D. 2014 & E.2013 & E.2014 & F.2014 \\
\hline \multirow[t]{6}{*}{ Actinobacteria } & & 0.18 & 0.33 & 2.00 & 0.38 & 2.04 & 0.22 & 2.17 & 0.17 & 0.31 & 0.02 & 0.40 \\
\hline & Arthrobacter & 0.01 & 0.001 & 0.19 & 0.02 & 0.07 & 0.001 & 0.004 & 0.02 & $<0.000$ & 0.001 & 0.17 \\
\hline & Kocuria & $<0.000$ & 0.01 & 0.01 & 0.004 & 0.69 & 0.04 & 0.001 & 0.002 & ND & ND & 0.001 \\
\hline & Corynebacterium & 0.004 & 0.01 & 0.53 & 0.18 & 0.08 & 0.08 & 0.04 & 0.02 & 0.02 & 0.005 & 0.05 \\
\hline & Pseudonocardia & 0.01 & 0.002 & 0.05 & 0.004 & 0.12 & ND & 0.39 & 0.004 & 0.02 & $<0.000$ & 0.001 \\
\hline & Rothia & 0.001 & 0.001 & 0.21 & 0.003 & 0.01 & 0.004 & 0.01 & 0.001 & 0.003 & 0.001 & 0.001 \\
\hline \multirow[t]{5}{*}{ Bacteroidetes } & & 24.15 & 2.52 & 1.00 & 0.93 & 0.95 & 1.37 & 0.81 & 0.05 & 0.60 & 0.70 & 0.78 \\
\hline & Chryseobacterium & 3.42 & 1.31 & 0.002 & 0.002 & 0.36 & 0.36 & 0.01 & 0.005 & 0.01 & 0.002 & 0.23 \\
\hline & Cloacibacterium & 0.02 & 0.002 & 0.21 & 0.004 & 0.19 & $<0.000$ & 0.64 & $<0.000$ & 0.09 & $<0.000$ & $<0.000$ \\
\hline & Flavobacterium & 3.00 & 0.31 & ND & 0.001 & 0.01 & 0.05 & 0.01 & 0.001 & 0.48 & 0.58 & 0.23 \\
\hline & Sphingobacterium & 17.34 & 0.70 & 0.16 & 0.69 & 0.33 & 0.88 & 0.004 & 0.01 & $<0.000$ & 0.02 & 0.11 \\
\hline \multirow[t]{8}{*}{ Firmicutes } & & 0.43 & 0.93 & 8.09 & 17.39 & 2.59 & 0.76 & 6.38 & 0.33 & 0.89 & 0.08 & 9.80 \\
\hline & Carnobacterium & ND & 0.002 & 0.002 & 0.003 & 0.002 & 0.004 & 0.001 & 0.01 & $<0.000$ & 0.002 & 7.32 \\
\hline & Enterococcus & 0.005 & 0.02 & 0.01 & 0.001 & 0.32 & 0.32 & 0.01 & $<0.000$ & 0.001 & ND & 0.002 \\
\hline & Lactobacillus & 0.03 & 0.21 & 0.04 & 3.16 & 0.05 & 0.01 & 0.02 & 0.01 & 0.003 & 0.002 & 0.003 \\
\hline & Lactococcus & 0.07 & 0.03 & 0.65 & 1.01 & 0.06 & 0.08 & 0.04 & ND & 0.01 & ND & 0.001 \\
\hline & Leuconostoc & 0.001 & 0.001 & 0.06 & 11.61 & 0.01 & 0.004 & 0.002 & ND & 0.001 & $<0.000$ & 0.02 \\
\hline & Streptococcus & 0.05 & 0.07 & 0.40 & 0.08 & 0.16 & 0.02 & 1.98 & 0.02 & 0.03 & 0.02 & 0.05 \\
\hline & Veillonella & 0.04 & 0.31 & 0.16 & 0.03 & 0.01 & 0.01 & 1.98 & 0.01 & 0.003 & 0.01 & 0.02 \\
\hline \multirow[t]{14}{*}{ Proteobacteria } & & 74.92 & 91.09 & 87.86 & 75.54 & 93.99 & 94.01 & 89.64 & 93.89 & 98.05 & 92.13 & 84.85 \\
\hline & Acinetobacter & 2.39 & 1.72 & 3.52 & 2.21 & 4.19 & 23.36 & 4.03 & 0.03 & 0.80 & 0.02 & 0.37 \\
\hline & Agrobacterium & 0.06 & 0.28 & 0.02 & 0.003 & 0.04 & 0.11 & 0.001 & 0.003 & $<0.000$ & 0.10 & 0.01 \\
\hline & Citrobacter & 0.27 & 0.07 & 0.002 & 0.03 & 5.95 & 3.75 & 0.01 & 0.01 & 0.001 & 0.02 & 14.00 \\
\hline & Cupriavidus & ND & 0.002 & ND & 6.57 & ND & 0.79 & ND & 86.96 & ND & 0.002 & 0.002 \\
\hline & Janthinobacterium & 2.57 & 0.001 & 0.001 & 0.002 & $<0.000$ & 0.07 & 0.003 & 0.03 & $<0.000$ & 3.83 & 3.78 \\
\hline & Mesorhizobium & 0.004 & ND & 0.04 & ND & 0.04 & ND & 0.08 & 0.001 & 0.02 & ND & $<0.000$ \\
\hline & Mycoplana & 0.14 & 0.17 & 0.001 & $<0.000$ & 0.001 & 0.7 & 0.01 & $<0.000$ & 0.001 & 0.01 & 0.001 \\
\hline & Ochrobactrum & 0.03 & 0.02 & 0.001 & 0.001 & 0.31 & 0.37 & ND & ND & ND & ND & $<0.000$ \\
\hline & Pseudomonas & 54.48 & 84.22 & 24.18 & 57.96 & 25.52 & 24.70 & 0.11 & 0.26 & 72.57 & 76.19 & 59.53 \\
\hline & Ralstonia & 4.18 & ND & 42.30 & ND & 28.65 & ND & 60.68 & 0.002 & 16.34 & $<0.000$ & 0.001 \\
\hline & Stenotrophomonas & 5.20 & 0.41 & 1.24 & 3.54 & 2.25 & 1.23 & 0.002 & 0.004 & ND & 0.02 & 0.004 \\
\hline & Sphingomonas & 0.03 & 0.07 & 0.49 & 0.20 & 0.23 & 0.18 & 0.57 & 0.31 & 0.06 & 0.07 & 0.04 \\
\hline & Yersinia & 0.01 & 0.001 & ND & 0.55 & 2.67 & 1.12 & ND & 0.002 & ND & 0.25 & 0.02 \\
\hline \multirow[t]{2}{*}{ Verrucomicrobia } & & 0.14 & 0.11 & 0.17 & 0.07 & 0.02 & 0.11 & 0.07 & 0.01 & 0.01 & 0.001 & 0.03 \\
\hline & Akkermansia & 0.001 & 0.01 & 0.09 & 0.02 & 0.01 & 0.002 & 0.01 & 0.01 & 0.001 & $<0.000$ & 0.02 \\
\hline
\end{tabular}

ND stands for non detected reads in the sample for that particular taxon 


\begin{tabular}{|c|c|c|c|c|c|c|}
\hline & Farm A & Farm B & Farm C & Farm D & Farm E & Farm $\mathbf{F}$ \\
\hline Farm area (ha) & 35 & 12 & 10 & 10 & 42 & 20 \\
\hline $\begin{array}{c}\text { Altitude above sea } \\
\text { level (m) }\end{array}$ & 194 & 1110 & 395 & 600 & 183 & 430 \\
\hline Jennies $^{\text {a }}$ (no.) & 45 & 40 & 40 & 70 & 32 & 32 \\
\hline Milking jennies a (no.) & $7-10$ & $7-10$ & $8-10$ & $30-33$ & $6-10$ & $6-10$ \\
\hline Herd breed & Crossbreds & Martina Franca & Crossbreds & Crossbreds & $\begin{array}{c}\text { Martina Franca, } \\
\text { Ragusana, } \\
\text { Crossbreds }\end{array}$ & Crossbreds \\
\hline Milking practice & $\begin{array}{l}\text { Automatic in } \\
\text { milking room }\end{array}$ & $\begin{array}{l}\text { Automatic in } \\
\text { milking room }\end{array}$ & $\begin{array}{c}\text { Automatic in } \\
\text { cowshed }\end{array}$ & Hand milking & $\begin{array}{l}\text { Automatic in } \\
\text { milking room }\end{array}$ & $\begin{array}{l}\text { Automatic in in } \\
\text { milking room }\end{array}$ \\
\hline Farming type & Semi-extensive & Semi-extensive & Semi-extensive & Extensive & Semi-extensive & Semi-extensive \\
\hline Feed & Grazing - Hay & $\begin{array}{l}\text { Hay - Bread - Protein } \\
\text { supplementation }\end{array}$ & Grazing - Hay & Grazing - Hay & Grazing - Hay & Grazing - Hay \\
\hline Milk use & Food - cosmetics & Food & Food - cosmetics & Food - cosmetics & Food - cosmetics & Cosmetics \\
\hline
\end{tabular}




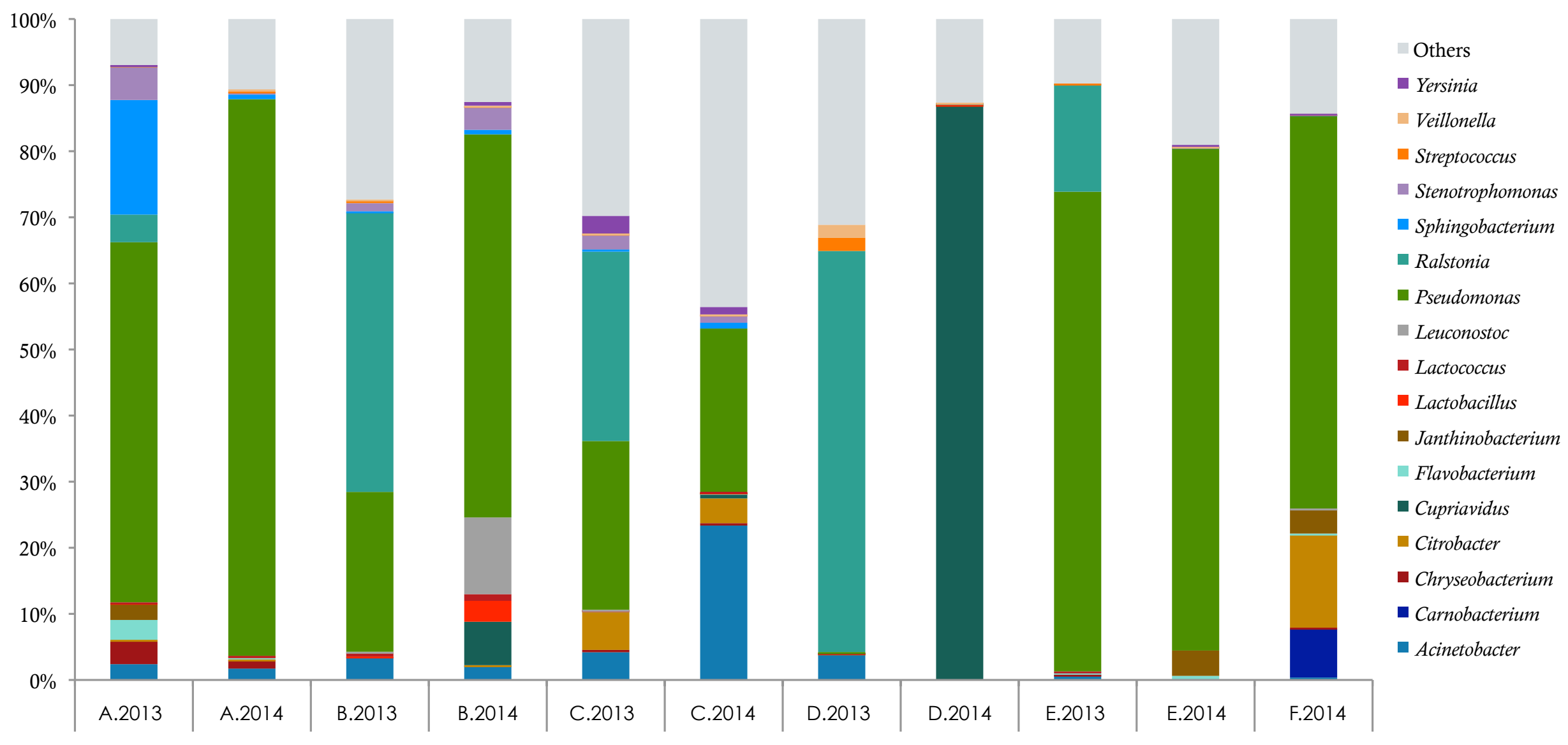


A)

Phylum

Actinobacteria

Bacteroidetes
Cyanobacteria

Firmicutes

Bacilli
Clostridia

Alphaproteobacteria

Proteobacteria

Betaproteobacteria

Gammaproteobacteria

Verrucomicrobiae

Order

Family

Corynecateriaceae

Micrococcaceae

Mycobacteriaceae

Nocardiopsaceae

Weeksellaceae

Shpingobacteriaceae

Sphingobacteriales

Streptophyta

Bacillales

Gemellales

Lactobacillales

Clostridiales

Rhizobiales

Sphingomonadales

Burkholderiales

Neisseriales

Enterobacteriales

Pasteurellales

Pseudomonadales

Verrucomicrobiales

Bacillaceae

Staphylococcaceae

Gemellaceae

Lactobacillaceae

Streptococcaceae

Veillonellaceae

Bradyrhizobiaceae

Methylobacteriaceae Phyllobacteriaceae

Rhizobiaceae

Sphingomonadaceae

Comamonadaceae

Oxalobacteraceae

Neisseriaceae

Enterobacteriaceae

Pasteurellaceae

Moraxellaceae

Pseudomonadaceae
B)

Genus

Actinomyces

Corynebacterium

Arthrobacter

Rothia

Mycobacterium

Cloacibacterium

Sphingobacterium

Bacillus

Staphylococcus

Lactobacillus

Streptococcus

Veillonella

Bradyrhizobium

Methylobacterium

Agrobacterium

Sphingomonas

Acidovorax

Janthinobacterium

Neisseria

Citrobacter

Escherichia

Acinetobacter

Pesudomonas
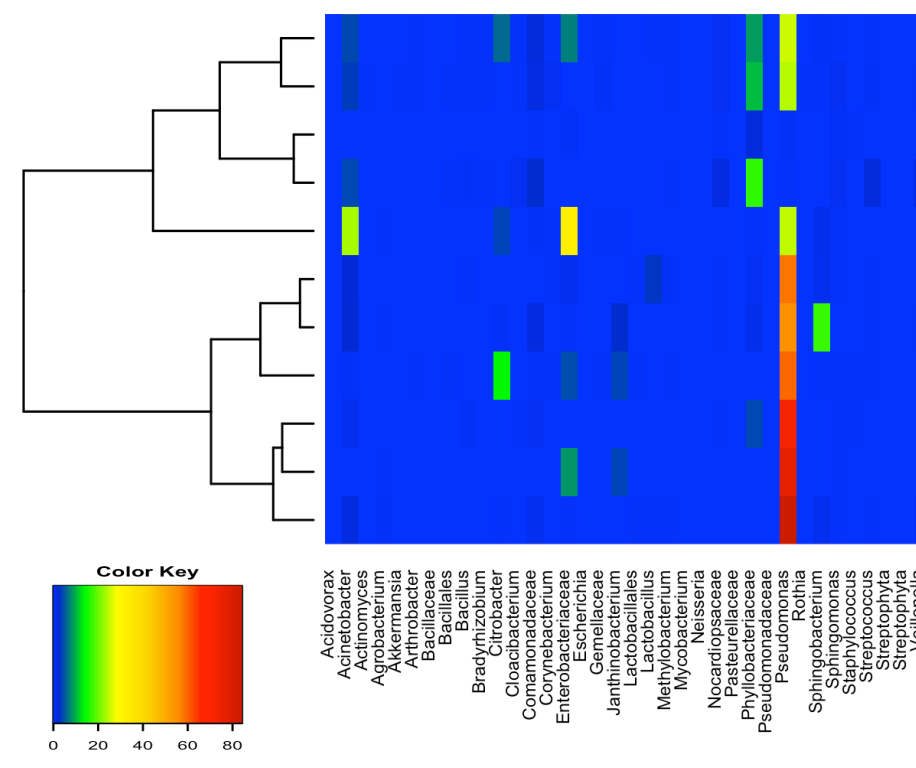

C. 2013

B.2013

D. 2014

D.2013

C. 2014

B.2014

A.2013

F.2014

E.2013

E.2014

A. 2014 
Figure 3

A)

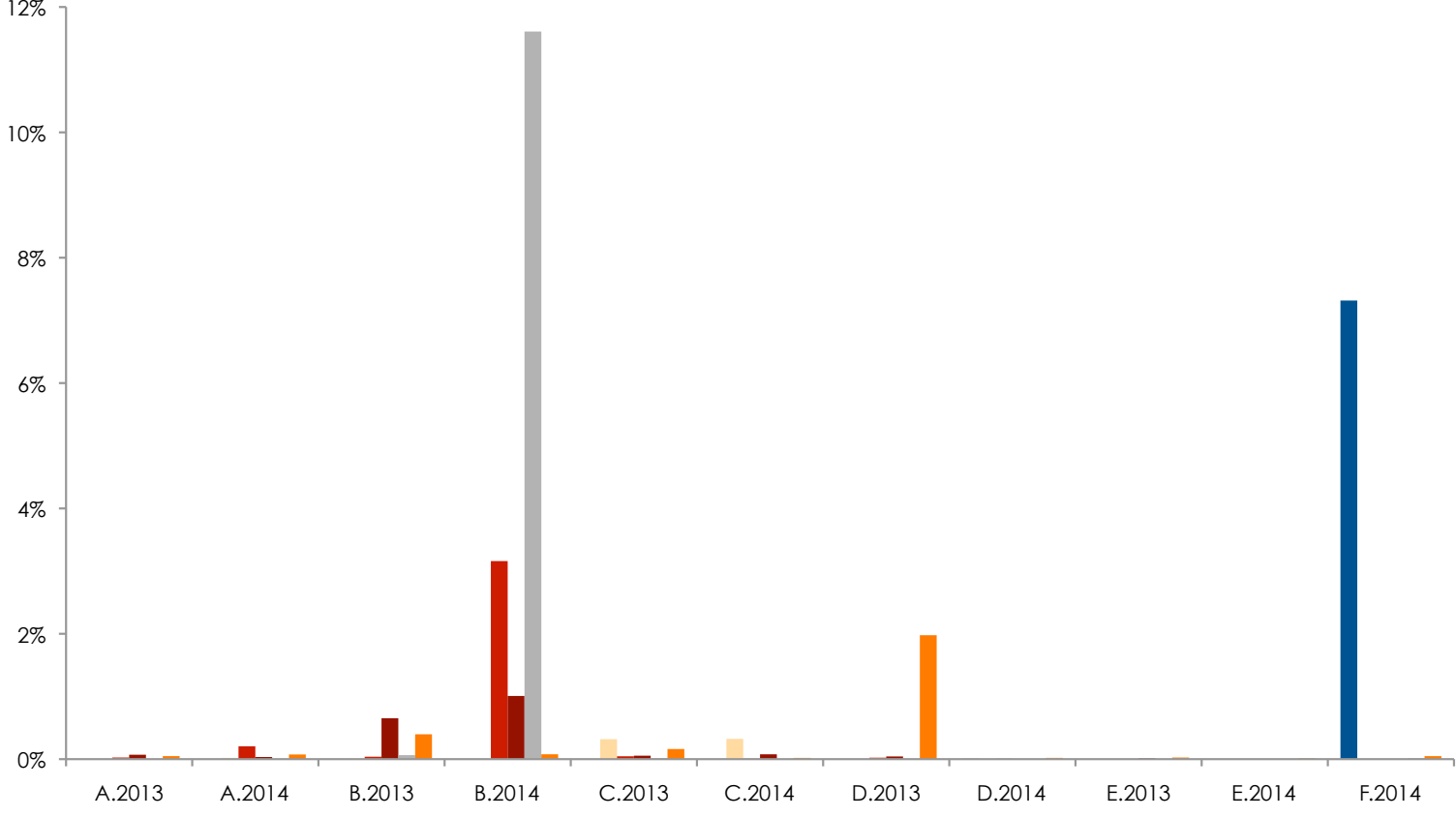

- Carnobacterium

Enterococcus

- Lactobacillus

- Lactococcus

- Leuconostoc

- Streptococcus

\section{C)}

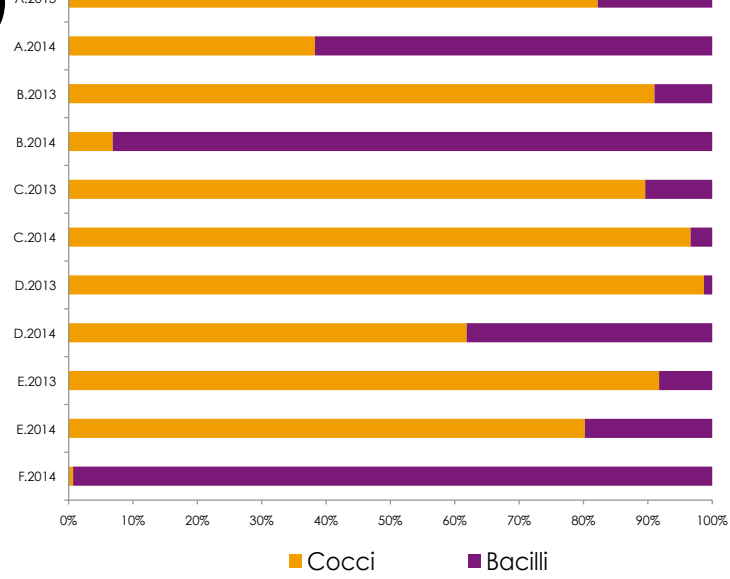

B)

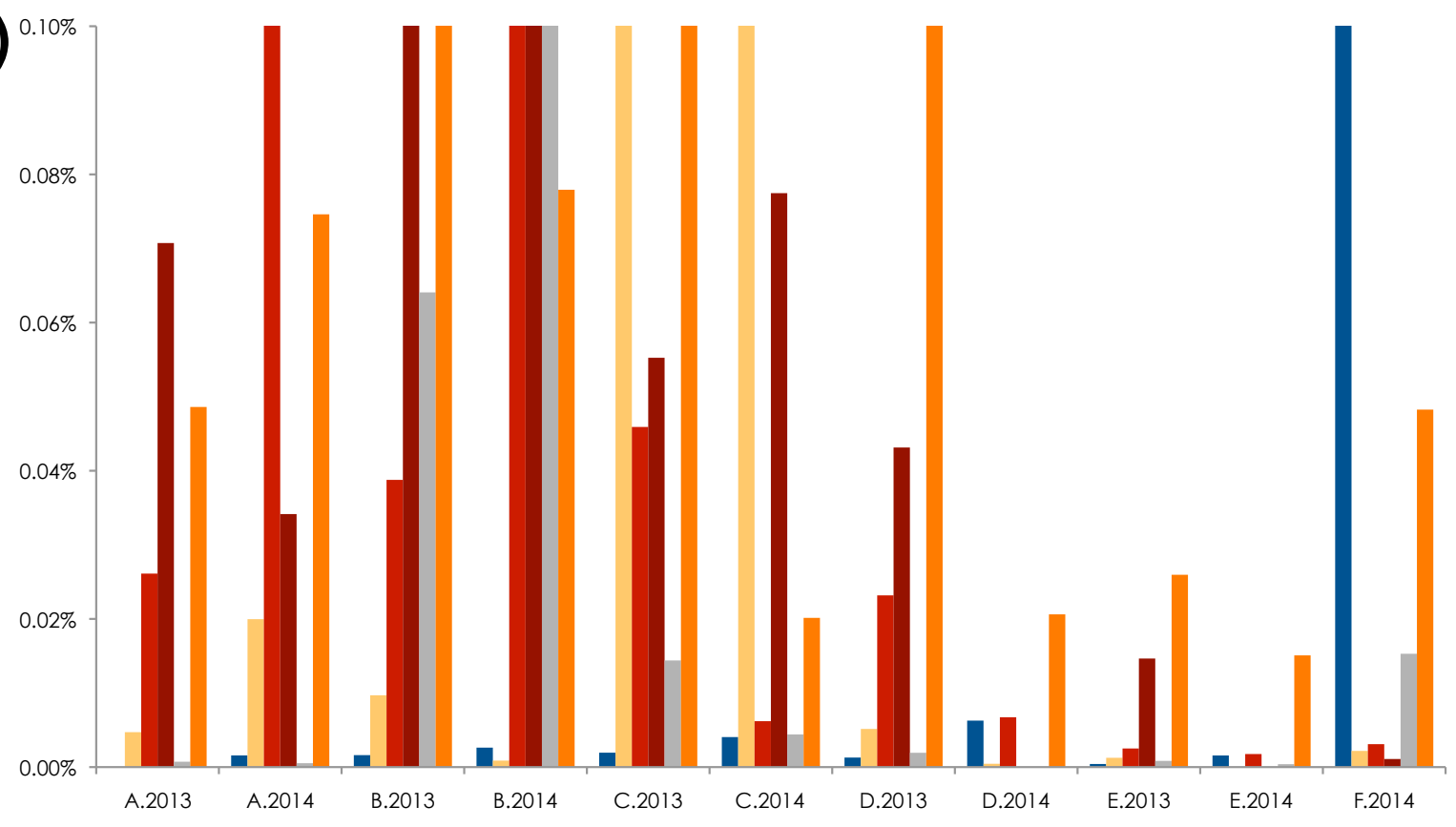

Carnobacterium

Enterococcus

- Lactobacillus

- Lactococcus

- Leuconostoc

- Streptococcus 


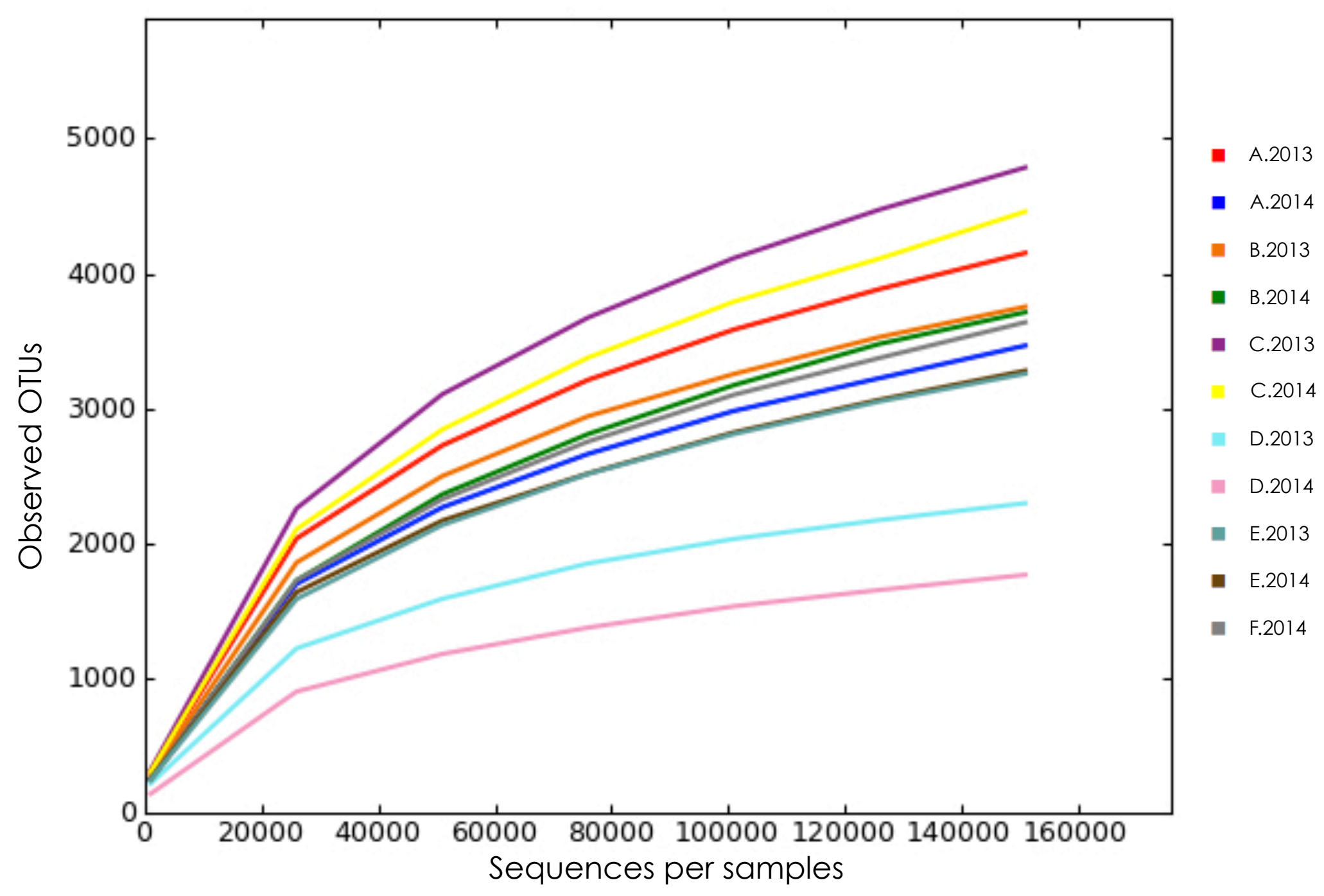




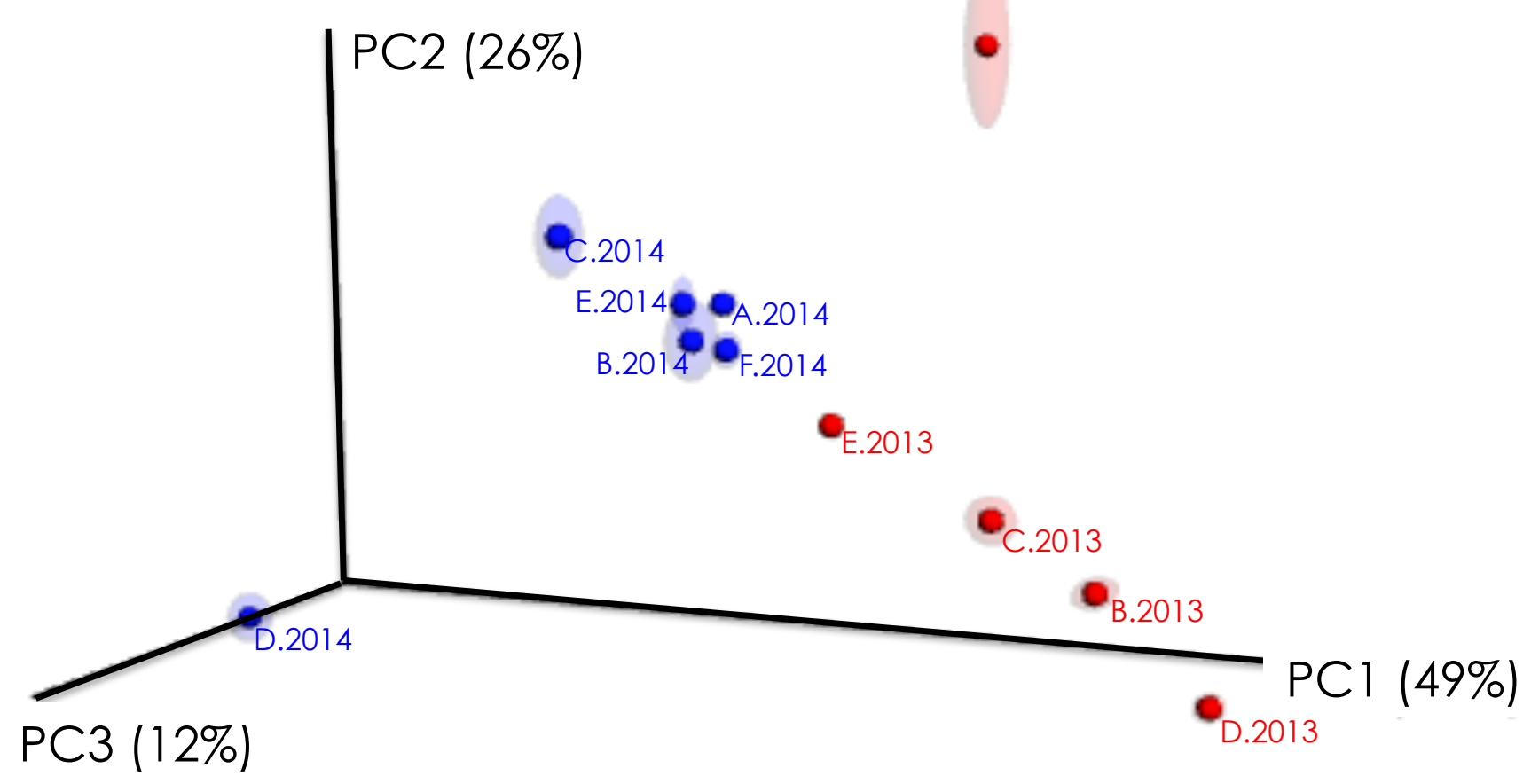

